\title{
The impact of COVID-19 on the lives and mental health of Australian adolescents
}

\author{
Sophie H. $\mathrm{Li}^{1}$. Joanne R. Beames ${ }^{1}$ · Jill M. Newby ${ }^{1,2} \cdot$ Kate Maston $^{1} \cdot$ Helen Christensen ${ }^{1} \cdot$ Aliza Werner-Seidler $^{1} \mathbb{C}$
}

Received: 30 March 2021 / Accepted: 15 April 2021 / Published online: 28 April 2021

(c) The Author(s) 2021

\begin{abstract}
There has been significant disruption to the lives and mental health of adolescents during the COVID-19 pandemic. The purpose of this study was to assess the psychological and lifestyle impact of the pandemic on Australian adolescents, using an online survey, administered during the outbreak. Self-report surveys were administered online to a sample of 760 Australian adolescents aged 12-18 years assessing impact on a range of domains including behaviour, education, relationships, lifestyle factors (exercise, technology use, and sleep), and mental health outcomes (psychological distress, loneliness, health anxiety and well-being). Results showed that three quarters of the sample experienced a worsening in mental health, since the pandemic began, with negative impacts reported on learning, friendships and family relationships. There were also high higher levels of sleep disturbance, psychological distress and health anxiety, relative to normative samples. Effects on mental health were worse among those who reported a previous diagnosis of depression and/or anxiety relative to those without no such history. Adolescents are already vulnerable to the onset of mental illness at this developmental stage, and the current research underscores the need to find rapid and accessible ways to support adolescent mental health during times of crisis.
\end{abstract}

Keywords Psychological distress $\cdot$ Depression $\cdot$ Anxiety $\cdot$ COVID-19 $\cdot$ Pandemic $\cdot$ Adolescent health

\section{Introduction}

As of the 11th April 2021, there have been more than 134 million COVID-19 cases and 2.9 million deaths worldwide [1]. With 29,369 cases and 909 deaths in Australia, even considering Australia's relatively small population size, the rate of COVID-19 infection and related deaths has remained low compared to many other countries [1]. As the pandemic and associated restrictions continue globally, there has been growing concern about the impact on mental health. Studies from around the world have shown that most individuals report increased psychological distress and worsened mental health [2-4], an effect which seems to be amplified among those with a history of mental illness [5, 6]. Although worsened mental health has been documented across the lifespan, several studies have found young adults (aged 18-24 years)

Aliza Werner-Seidler

a.werner-seidler@blackdog.org.au

1 Black Dog Institute, UNSW, Hospital Road, Randwick, Sydney 2022, Australia

2 School of Psychology, UNSW, Sydney, NSW, Australia are experiencing the greatest deterioration in mental health $[3,7]$. Very few studies have assessed the impact of the pandemic-related disruption on adolescents [8], which is concerning, because adolescence represents a time of social transformation, marked by an increased need for peer interaction and heightened sensitivity to social stimuli [9]. Adolescents demonstrate increased independence from their families, begin to establish relationships with peers based on shared values and ideas [10], and become more sensitive to peer acceptance, approval and rejection than either children or adults [11]. Because of this sensitivity, it is likely that social distancing measures, together with school closures, may have a greater negative effect on adolescents. Support for this comes from a recent review of the literature involving more than 50,000 young people that found social isolation and loneliness significantly increases the risk of mental illness in young people [12].

Despite the significant potential impact of pandemicrelated restrictions on adolescents, little empirical work has addressed this possibility [13]. Six published studies have assessed adolescent mental health $(<18$ years $)$ in response to the pandemic, three conducted in China, one each in Canada, Germany and the US [14-19], which 
collectively show that the prevalence of mental illness (most commonly depression and anxiety) is elevated relative to pre-pandemic prevalence estimates. Evidence of a similar pattern of worsening youth mental health in Australia is emerging. For example, the Kids Helpline (a 24-h free counselling service for 5-25 years), has reported a $40 \%$ increase in calls to the service, and the number of young people attending the hospital Emergency Department for self-harm has increased by $33 \%$, both relative to the same period in 2019 [20, 21]. In addition, young people's behavior and lifestyles have also been impacted by COVID-19. For example, one study [17] found that most teenagers reported engaging in at least some social distancing, and another found that $94 \%$ of adolescents reported engaging in protective behaviours, such as wearing a mask, hand washing and social distancing [15]. Moreover, worsening sleep and feelings of isolation and loneliness have been reported [22], as has disruption to learning and education [15]. Together, the available data indicates significant impact on young people's daily lives.

Beyond these studies, it is unknown how the disruption to daily life has impacted adolescents' behavior and worries as they relate to COVID-19. The impact on their peer relationships, family relationships, feelings of loneliness, learning and education, lifestyle factors (e.g. exercise, sleep, and technology use), and how these relate to mental health, requires investigation to understand the support adolescents require. The overall objective of the current study is to address this gap and answer calls from the scientific community to assess how young people's lives and mental health has been impacted by the pandemic [23, 24]. The first aim of this study was to investigate the psychological impact of the pandemic on adolescents. We included measures of psychological distress, loneliness, health anxiety and wellbeing, which we expected to have worsened, as informed by the emerging literature [14, 15, 17]. Based on previous studies from the adult literature $[5,6]$, we expected that those who had a pre-existing history of depression and/or anxiety would show a worse psychological response to the pandemic.

The second aim was to understand how the pandemic and associated containment measures impacted the lives of adolescents. To this end, we collected data on young people's demographic characteristics, worry about contracting COVID-19, changes to behavior, sleep disturbance, exercise, and technology use. Consistent with past studies, we expected young people to express significant concerns about contracting COVID-19, and to report changes in behavior, sleep patterns, exercise and technology use. A final exploratory aim was to examine the relationship between levels of worry about pandemic, behaviour change, uncertainty about the future, exercise, technology use, sleep, loneliness, wellbeing, psychological distress and health anxiety.
Data was collected from an online survey distributed via social media advertisements. While this method may not produce a representative sample of the entire population [25], it nonetheless allowed timely access to a significant number of young people, while containment measures remained in place. The recruitment period (end of June-beginning of August 2020) included the relaxing of lockdown restrictions across Australia (end of June-beginning of July), with the exception of Melbourne and the state of Victoria, which went into a second lockdown on the 8th of July (Melbourne) and 2nd of August (Victoria). As a result of these restriction differences, we also compared young people in Victoria who completed the survey during the second lockdown (on or after 8th July) to young people in the rest of the country on mental health outcomes. At the time of survey completion (31st of August 2020), there had been over 24 million COVID-19 cases and 838, 924 deaths worldwide, with 25, 547 cases and 600 deaths in Australia.

\section{Method}

\section{Participants}

Participants were aged 12-18 years, living in Australia. Data was collected via the Qualtrics platform from 22 June 2020 to 5 August 2020.

\section{Ethics approval and consent}

The study was approved by the UNSW Human Research Ethics Committee (HC200334). All respondents were required to pass a Gillick Competency Task to ensure they understood the study, and had the capacity to provide informed consent, before providing consent [26].

\section{Measures}

\section{Demographics, general health and mental health history}

Information was collected on participants' age, school grade, gender, country of birth, language spoken at home, Aboriginal and Torres Strait Islander status, state of residence and who they lived with. Participants indicated if they had a parent or carer whose job had been impacted by the pandemic. Participants rated their self-rated health [27], and were asked whether they had a chronic illness, had ever been diagnosed with depression or anxiety by a professional, and current mental health treatments. 


\section{COVID-19 exposure, perceived risk and behaviour change}

The items below were generated from a previous survey [28].

- COVID-19 exposure: participants were asked five yes/ no questions about COVID-19-whether they had been tested/diagnosed, whether a family member or close contact had been diagnosed, and whether they had been required to quarantine for 14 days.

- Perceived risk: participants were asked four questions relating to their perceptions of risk and their concerns about contracting COVID-19. The first question assessed how worried they were about catching COVID-19 on a five-point scale (not at all-extremely concerned). They then rated perceived likelihood they would catch the virus on a visual analogue scale (VAS) from 0 (not at all likely) to 100 (extremely likely), and perceived behavioural control (i.e., how much they thought they could do to protect themselves from catching the virus), on a 0 (I can't do anything) to 100 (I can do a lot) VAS. The final questions assessed perceived illness severity if they did catch COVID-19 (response options were: no symptoms, mild symptoms, moderate symptoms, severe symptoms, severe symptoms requiring hospitalisation, and severe symptoms leading to death).

- Behaviour change: Participants indicated whether they had engaged in eight social distancing and hygiene behaviours over the past week (hand washing, hand sanitising, facemask wearing, avoidance of: going to the shops/touching surfaces/spending time with people from outside the household/going to school and whether they stayed home as much as possible). Response options were on a five-point scale $(1=$ not at all to $5=$ to all of the time).

\section{COVID-19 impact on physical and mental health, school/ education and relationships}

- Physical and mental health: young people were asked, in two separate questions, whether their physical and mental health had been impacted by the pandemic (a lot better, a little better, stayed the same, a little worse, a lot worse).

- School and education: respondents indicated whether their school shut down during the pandemic and whether they participated in online learning (both yes/no). Those engaged in online learning reported up to three challenging aspects of completing school work at home, from a forced choice list (not enough family support, parents working and didn't have time to help, access to technology, speed of internet, harder to learn online, too many distractions, not enough support from teachers, not motivated, other). Participants indicated how much they felt the pandemic had impacted their learning overall (positively, not at all, and negatively).

- Peer relationships: two questions assessed how the pandemic had impacted participants' friendships; first, how socially connected they felt to their friends (more connected, no change, less connected); and second, impact on friendships overall (positively, negatively, and not at all).

- Family functioning: two items assessed young people's family relationships, in terms of overall impact on relationships with family members (improved, no change, worsened), and impact on family stress levels (less stress, no stress, more stress).

\section{Lifestyle factors}

- Exercise: participants indicated the number of days they exercised for at least $30 \mathrm{~min}$ over the previous week (0, 1-2 days, 2-4 days, 5-6 days, every day, and don't know), and to report whether, they exercised more, less, or the same amount as they usually would.

- Technology use: for the previous week, participants estimated total time spent in front of screens each day, not including time spent on school work $(<1 \mathrm{~h}, 1-2 \mathrm{~h}$, 2-4 h, 6-8 h, and $>8$ h). They also reported how much time was used to connect with friends or family (same response options) and whether their use of technology had changed since the pandemic (less than before, the same, more than before).

- Sleep: sleep was measured using the Insomnia Severity Index (ISI), which is a seven-item self-report measure of insomnia symptoms over the previous 2 weeks [29]. Responses are reported on a Likert scale from 0 to 4 with the following cut-off scores: $0-7$ no clinically significant insomnia, 8-14 subthreshold insomnia, 15-21 moderate severity insomnia, and 22-28 severe insomnia [29]. The ISI has been widely administered to, and validated in, general adolescent samples [30].

- Loneliness: a single item was selected to assess loneliness and was taken from the UCLA Loneliness Scale [31]. Participants were asked to indicate how often they felt alone over the past 2 weeks (hardly ever, some of the time, and often).

- Uncertainty about the future: a single item was selected to ask participants about their feelings of uncertainty about the future, ranging from 'not at all' to 'extremely' on a five-point scale, where a higher score represents greater levels of uncertainty.

\section{Mental health and well-being}

The Kessler-6 (K6) assessed general psychological distress over the past 30 days $[32,33]$. The K6 is a valid and reliable 
predictor of probable mental illness. Clinical validation studies, which define mental illness as the presence of a DSM disorder, have found the K6 has a specificity of 0.96 and a total accuracy of 0.92 , and has comparable psychometric properties to clinical tools, such as the Compositive International Diagnostic Inventory in assessing the presence of probable mental illness [33]. The K6 has been validated and used in the largest population mental health survey in Australia [34] and has also been validated in adolescent samples [35], making it ideally suited to measure adolescent psychopathology in large surveys.

The seven-item Warwick Edinburgh Mental Well-Being Scale-short form (SWEMWS; [36]) assessed mental wellbeing over the past 2 weeks and has validated for use in young people [37]. The three-item Body Preoccupation Scale of the Illness Attitude Scales [38] was administered to assess health anxiety.

\section{Procedure}

Participants were recruited via study information posted on the Black Dog Institute website and via paid advertisements circulated on Facebook and Instagram which targeted 12-18-year-old Australians. Participants responded to advertisements by clicking on a link which took them to the survey landing page. They read the electronic information sheet and consent form, completed the Gillick Competency Task, before accessing the survey. Upon completion, participants were placed in a draw to win one of five $\$ 50$ vouchers.

\section{Statistical analyses}

Demographic and clinical characteristics were reported using descriptive analyses. Where possible, outcomes from standardised measures were compared to normative, general population data. Independent samples $t$ tests compared participants with and without a prior diagnosis of anxiety and/ or depression on outcome variables (e.g. K6). Zero order correlations were conducted between worry about COVID, behaviour change, lifestyle and mental health variables.

\section{Results}

In total, 1743 young people viewed the study page, and 945 participants provided consent and started the survey. Of those, 185 did not provide enough data $(<90 \%$ complete) to be included. The final sample comprised 760 participants.

\section{Demographics}

Table 1 summarizes participant characteristics. Participants were 14.8 years on average, and ranged in school year from
Table 1 Participant characteristics, clinical history and COVID-19 exposure

\begin{tabular}{|c|c|}
\hline Participant Characteristics & $N(\%)$ \\
\hline \multicolumn{2}{|l|}{ Age } \\
\hline Age in years, mean (SD, range) & $14.80(1.26,12-18)$ \\
\hline 12 & $31(4.1)$ \\
\hline 13 & $101(13.4)$ \\
\hline 14 & $154(20.4)$ \\
\hline 15 & $214(28.3)$ \\
\hline 16 & $225(29.8)$ \\
\hline 17 & $22(2.9)$ \\
\hline 18 & $9(1.2)$ \\
\hline \multicolumn{2}{|l|}{ Gender } \\
\hline Male & 144 (19) \\
\hline Female & $544(72)$ \\
\hline Non-binary & $38(5)$ \\
\hline Different identity & $14(1.9)$ \\
\hline Prefer not to say & $16(2.1)$ \\
\hline \multicolumn{2}{|l|}{ State } \\
\hline New South Wales & $238(31.5)$ \\
\hline Victoria & $266(35.2)$ \\
\hline Queensland & $116(15.3)$ \\
\hline South Australia & $42(5.6)$ \\
\hline Western Australia & $53(7)$ \\
\hline Tasmania & $20(2.6)$ \\
\hline Australian capital territory & $18(2.4)$ \\
\hline Northern territory & $1(0.1)$ \\
\hline \multicolumn{2}{|l|}{ Living situation } \\
\hline Dual parent family & $480(63.5)$ \\
\hline Single parent family & $151(20)$ \\
\hline Blended family & $98(13)$ \\
\hline $\begin{array}{l}\text { Other (living with grandparents, sibling, } \\
\text { other) }\end{array}$ & $27(3.6)$ \\
\hline \multicolumn{2}{|l|}{ Place of birth } \\
\hline Born in Australia & $666(88.1)$ \\
\hline \multicolumn{2}{|l|}{ English main language spoken at home } \\
\hline Yes & $663(87.7)$ \\
\hline \multicolumn{2}{|l|}{ Aboriginal or Torres Strait Islander } \\
\hline Yes & $71(9.4)$ \\
\hline \multicolumn{2}{|l|}{ General health } \\
\hline Excellent & $103(13.6)$ \\
\hline Very good & $291(38.5)$ \\
\hline Good & $225(29.8)$ \\
\hline Fair & $111(14.7)$ \\
\hline Poor & $26(3.4)$ \\
\hline \multicolumn{2}{|l|}{ Current chronic illness status } \\
\hline Yes & $159(21)$ \\
\hline No & $532(70.4)$ \\
\hline Unsure & $54(7.1)$ \\
\hline Prefer not to say & $11(1.5)$ \\
\hline \multicolumn{2}{|l|}{ Diagnosis of anxiety or depression } \\
\hline Yes, depression only & $26(3.4)$ \\
\hline
\end{tabular}


Table 1 (continued)

\begin{tabular}{lc}
\hline Participant Characteristics & $N(\%)$ \\
\hline Yes, anxiety only & $84(11.1)$ \\
Yes, depression and anxiety & $155(20.5)$ \\
No & $401(53)$ \\
Unsure & $50(6.6)$ \\
Prefer not to say & $40(5.3)$ \\
Current treatment for mental health problem & \\
Yes & $210(27.8)$ \\
No & $501(66.3)$ \\
Unsure & $14(1.9)$ \\
Prefer not to say & $31(4.1)$ \\
COVID Exposure & \\
Contracted COVID-19 & $2(0.3)$ \\
Tested for COVID-19 & $112(14.7)$ \\
Family member who contracted COVID-19 & $14(1.9)$ \\
Close contact who contracted COVID-19 & $70(9.2)$ \\
Required to quarantine or self-isolate for & $85(11.2)$ \\
14 days & \\
\hline
\end{tabular}

years 7-12. Most were female (72\%), spoke English at home (87.7\%) and were born in Australia (88.1\%). Participants lived across all states and territories. Almost two-thirds of the sample (63.2\%) reported living with two parents, and $50 \%$ indicated that their parent or career's job had been impacted by the pandemic. General population data from the largest, most recent national mental health survey, the Australian Child and Adolescent Survey of Mental Health [39], indicated comparable demographic characteristics in a randomly selected sample of 6310 families. Specifically, in that survey, $87.8 \%$ were born in Australia and 68.6\% lived with two parents or carers from their original family. Data on language spoken at home was not collected.

\section{General health and mental health history}

The mean rating for overall health was 3.44/5 $(\mathrm{SD}=1.01)$, with most rating their health as either 'good' $(29.8 \%)$ or 'very good' (38.5\%). Twenty one percent reported a current chronic illness, 34\% reported a previous diagnosis of either depression or anxiety, and $27.8 \%$ were receiving current mental health treatment (see Table 1).

\section{COVID exposure, perceived risk and behavior change}

- Exposure: only $0.3 \%$ had a diagnosis of COVID-19. Few participants had a family member who had contracted the virus (1.9\%), while just under $10 \%$ had a close contact who had the virus.
- Perceived risk: on average, young people expressed moderate levels of worry about contracting the virus $(M=2.27, \mathrm{SD}=0.97)$, and most $(68.5 \%)$ were 'a little concerned' or 'moderately concerned' about catching the virus. Respondents thought it was relatively unlikely that they would contract COVID-19 $(M=25.29 ; \mathrm{SD}=19.81$; scale $0-100)$, and reported a sense of agency that their behaviors could prevent them contracting the infection $(M=60.75 ; \mathrm{SD}=24.29$; scale $0-100)$. Young people expected if they contracted the virus they would experience either no $(5.3 \%)$ or mild $(39.8 \%)$ symptoms, with $37.2 \%$ expecting moderate symptoms.

- Behavior change: most engaged in protective healthrelated behaviours over the previous week. Reports of handwashing were high, with $68.6 \%$ indicating they washed their hands thoroughly 'all of the time' or 'most of the time' as was sanitizer use $(72.1 \%$ all or most of the time). Most (85.6\%) respondents avoided touching objects or surfaces touched by other people to at least some extent, and $30 \%$ reported wearing a facemask. Respondents also avoided places to reduce the spread, with $82.1 \%$ avoiding the shops at least some of the time (43.3\% reported avoiding shops all or most of the time), $81.3 \%$ avoiding leaving the house ( $48.4 \%$ always or most of the time), and $70.4 \%$ avoiding spending time with people outside their household $(35.1 \%$ always or most of the time). Half reported not going to school in the previous week due to the pandemic.

\section{COVID-19 impact}

- Physical and mental health: see Fig. 1. More than half of the participants indicated that their physical health had worsened during the pandemic. Approximately a third reported no change, and few reported an improvement. The impact of the pandemic on mental health revealed different results; most (75\%) young people reported a negative effect on their mental health. Few reported no change or an improvement.

- School and education: most (87\%) reported that their schools closed during the pandemic, and the majority (95.1\%) engaged in online learning instead. The top three challenges to online learning were: (i) a lack of motivation; (ii) too many distractions at home; and (iii) it being more difficult to learn via online platform relative to faceto-face. A lack of support from schoolteachers, increased school workload, feelings of loneliness and slow internet was also noted. Overall, two in three young people $(62.6 \%)$ felt that the pandemic had negatively impacted their learning, with $22 \%$ indicating no change and $14.9 \%$ reporting a positive impact.

- Peer relationships: see Fig. 2. Most respondents reported feeling less connected to their friends. There seemed to 
Fig. 1 Physical and mental health change since the pandemic began
Physical Health Change

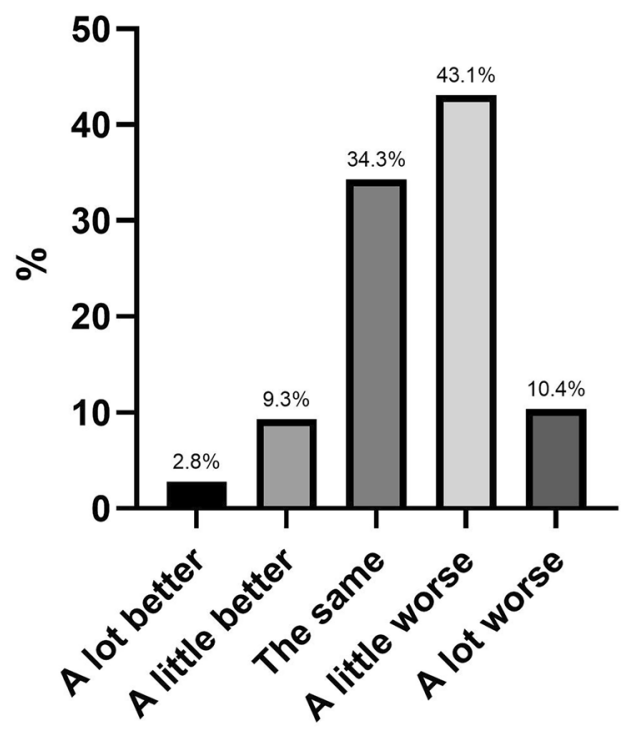

Mental Health Change

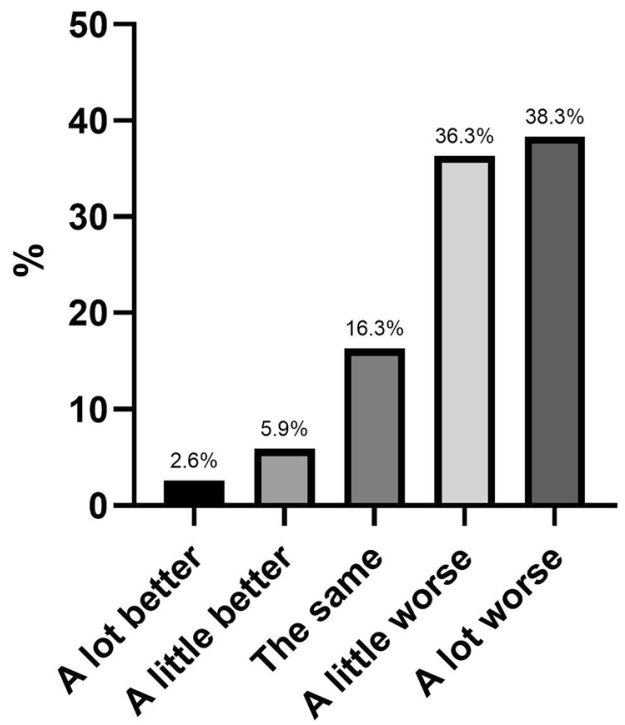

be a degree of stability to adolescents' friendships, with about half of the sample reporting no overall impact on their friendships.

- Family functioning: see Fig. 2. Approximately one third of respondents reported a worsening of family relationships, and most young people reported a worsening of family stress.

\section{Lifestyle factors}

See Table 2 .

- Exercise: twenty percent reported zero instances of at least 30-min exercise during the previous week. Approximately half of respondents indicated that they exercised for $30 \mathrm{~min}$ on at least 1-2 days. Most young people reported either a decrease in exercise, since the pandemic began, or no change.

- Technology use: most young people reported either 2-4 h or 4-6 h daily screen use. There was a still a significant proportion of young people reporting higher levels of use ( $>8 \mathrm{~h}$ ) each day, with $72 \%$ reporting increased technology use to connect with others, usually spending around 4-h online for interaction. Almost three quarters of the sample reported increased technology use since the start of the pandemic.

- Sleep disturbance: the mean score on the ISI was 12.09 $(\mathrm{SD}=6.03)$. Just under half of the sample $(40.5 \%)$ reported subthreshold insomnia, while more than a quarter (28.7\%) reported moderate severity insomnia and a further $6.4 \%$ reporting severe insomnia.

- Loneliness: Just over half (51.4\%) of the sample reported frequently experiencing feelings of loneliness. A further third reported feeling alone some of the time.

- Uncertainty about the future: ninety-three percent of respondents reported some degree of uncertainty about the future, with approximately one third (33.8\%) reporting very or extreme levels of uncertainty.

\section{Mental health and well-being}

- Mental health: The mean score on the K6 was 18.08 ( $\mathrm{SD}=6.63$; range 6-30), with just under half of the sample (48.3\%) scoring above the threshold that indicates psychological distress indicative of mental illness.

- Well-being: Mean well-being score was 18.79 ( $\mathrm{SD}=5.87$; range 7-35), with a higher score indicating greater levels of well-being.

- Health anxiety: on the screening survey for health anxiety, the Body Preoccupation Scale of the Illness Attitude Scales, $40.1 \%$ of young people scored above the clinical cutoff which indicates severe health anxiety. 
Fig. 2 Impact of pandemic on peer and family relationships
Social Connection

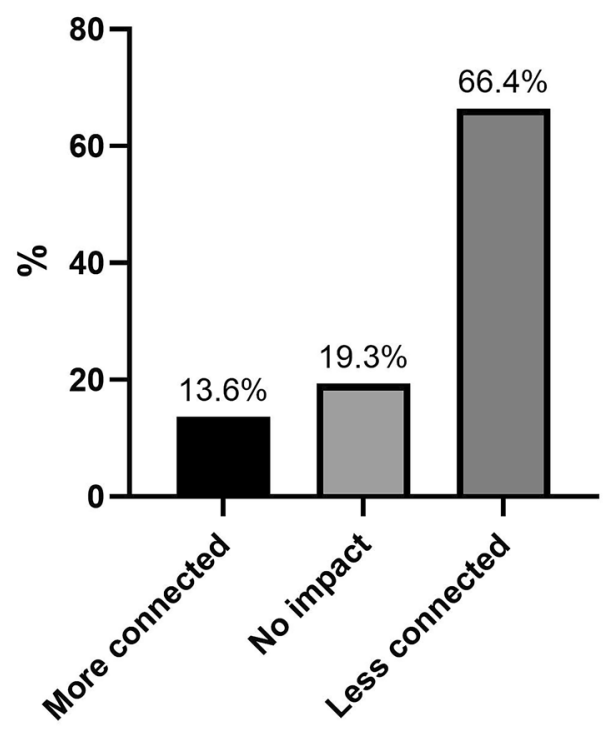

Family Relationships

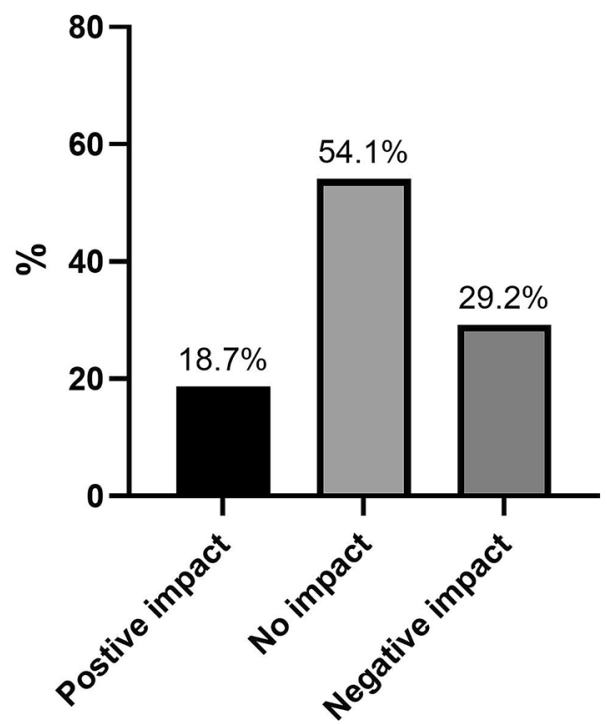

Friendships

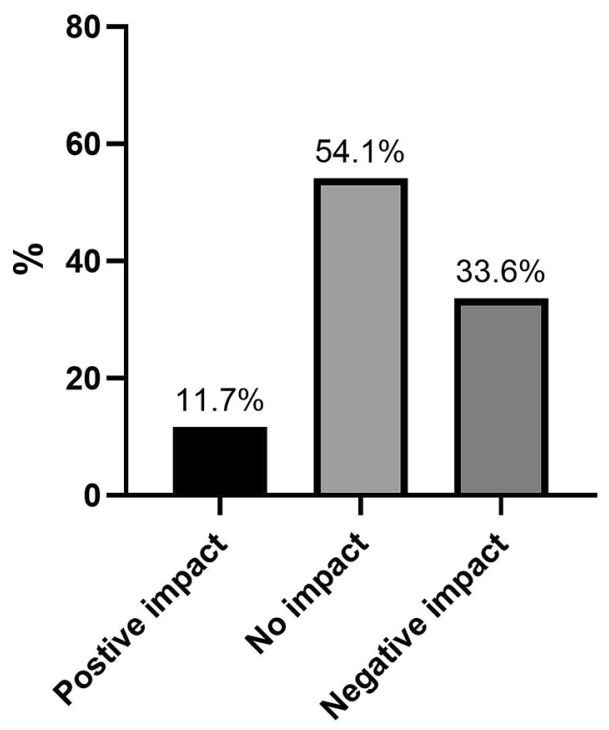

Family Stress

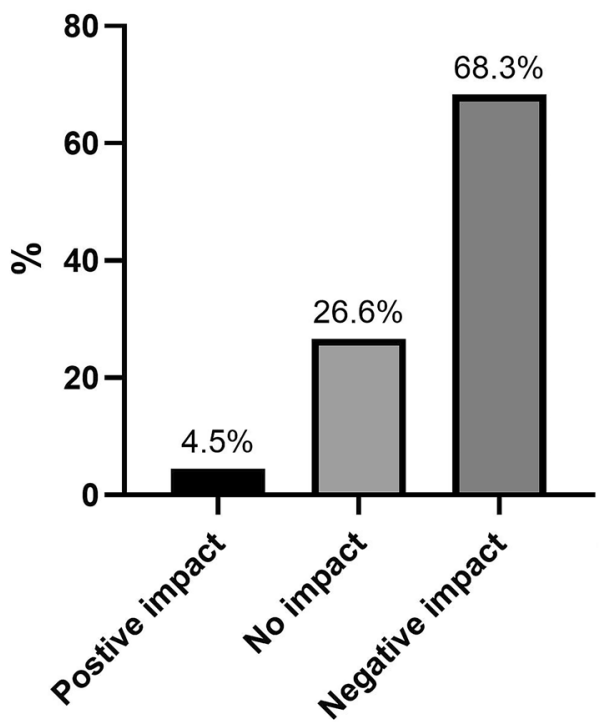

\section{Comparison of mental health outcomes}

between young people in Victoria during the second lockdown and young people from the rest of Australia

Given that a second lockdown in Victoria was announced on the 8th of July, mid-way during the survey data collection period, we examined the mental health outcomes among young people located in this state who responded to the survey on or after this date $(n=116)$, relative to respondents across the rest of Australia, or in Victoria prior to this date $(n=514)$. We found no significant differences in mental health outcomes including loneliness, $[t(628)=-1.85$, $p>0.05]$, psychological distress $(t<1)$, well-being $(t<1)$, or health anxiety, $(t<1)$. Accordingly, data was collapsed and not segregated by region for the analysis.

\section{Comparison between adolescents} with and without a prior mental health diagnosis

Young people with and without a self-reported diagnosis of depression and/or anxiety were compared on outcomes (see Table 3). After removing those who did not know if they had a diagnosis ( $6 \%$ of the overall participant pool) or chose not to say $(5 \%), 60 \%$ percent of the remaining sample reported no history of mental illness, and $40 \%$ reported a previous 
Table 2 Frequency and descriptive data for lifestyle and mental health outcomes

\begin{tabular}{|c|c|c|}
\hline \multicolumn{2}{|l|}{ Lifestyle and Mental Health Outcomes } & $N(\%)$ \\
\hline \multicolumn{3}{|l|}{ Exercise (\# $\geq 30$ min exercise sessions in past week) } \\
\hline None & & $154(20.3)$ \\
\hline $1-2$ days & & $236(31.1)$ \\
\hline $3-4$ days & & $169(22.2)$ \\
\hline 5-6 days & & $102(13.4)$ \\
\hline $7+$ days & & $76(10)$ \\
\hline \multicolumn{3}{|l|}{ Exercise change due to the pandemic } \\
\hline Less than usual & & $308(40.5)$ \\
\hline Same as usual & & $326(42.9)$ \\
\hline More than usual & & $121(15.9)$ \\
\hline \multicolumn{3}{|l|}{$\begin{array}{l}\text { Technology use } \\
\text { (time spent on screens each day for non-school purposes) }\end{array}$} \\
\hline$<1 \mathrm{~h}$ & & $11(1.4)$ \\
\hline $1-2 \mathrm{~h}$ & & $33(4.3)$ \\
\hline $2-4 \mathrm{~h}$ & & $178(23.4)$ \\
\hline $4-6 \mathrm{~h}$ & & $241(31.7)$ \\
\hline $6-8 \mathrm{~h}$ & & $151(19.9)$ \\
\hline$>8 \mathrm{~h}$ & & $141(18.6)$ \\
\hline \multicolumn{3}{|l|}{$\begin{array}{l}\text { Technology use-social connection } \\
\text { (amount of time spent connecting with friends or family) }\end{array}$} \\
\hline$<1 \mathrm{~h}$ & & $173(22.8)$ \\
\hline $1-2 \mathrm{~h}$ & & $243(32)$ \\
\hline $2-4 \mathrm{~h}$ & & $196(25.8)$ \\
\hline $4-6 \mathrm{~h}$ & & $96(12.6)$ \\
\hline $6-8 \mathrm{~h}$ & & $23(3)$ \\
\hline$>8 \mathrm{~h}$ & & $24(3.2)$ \\
\hline \multicolumn{3}{|l|}{ Technology use change due to the pandemic } \\
\hline Less than usual & & $81(10.7)$ \\
\hline Same as usual & & $128(16.8)$ \\
\hline More than usual & & $547(72)$ \\
\hline \multicolumn{3}{|l|}{ Loneliness (frequency of feeling alone) } \\
\hline Hardly ever & & $130(17.1)$ \\
\hline Some of the time & & $233(30.7)$ \\
\hline Often & & $391(51.4)$ \\
\hline \multicolumn{3}{|l|}{ Uncertainty about the future } \\
\hline Not at all & & $54(7.1)$ \\
\hline A little uncertain & & $218(28.7)$ \\
\hline Moderately uncertain & & $227(29.9)$ \\
\hline Very uncertain & & $158(20.8)$ \\
\hline Extremely uncertain & & $99(13)$ \\
\hline Standardised questionnaires & $M(\mathrm{SD})$ & Range \\
\hline Insomnia Severity Index & $12.09(6.03)$ & $0-28$ \\
\hline Short Warwick-Edinburgh Mental Well-being Scale & $18.97(5.87)$ & $7-35$ \\
\hline Kessler-6 Scale (psychological distress) & $18.08(6.63)$ & $6-30$ \\
\hline Illness Preoccupation Scale (health anxiety) & $4.72(4.72)$ & $0-12$ \\
\hline
\end{tabular}

diagnosis of depression or anxiety (3.9\% depression only, $12.6 \%$ anxiety only, and $23.3 \%$ both depression and anxiety). These categories were collapsed for the subsequent analyses into presence or absence of a diagnosed depression and/or anxiety. Participants with a history of depression or anxiety reported worse mental and physical health on all measures 
Table 3 Comparison between respondents with and without a self-reported mental health diagnosis of depression and/or anxiety

\begin{tabular}{|c|c|c|c|c|c|c|c|}
\hline & \multicolumn{3}{|c|}{$\begin{array}{l}\text { Prior mental health } \\
\text { diagnosis }\end{array}$} & \multicolumn{3}{|c|}{$\begin{array}{l}\text { No prior mental } \\
\text { health diagnosis }\end{array}$} & \multirow[t]{2}{*}{ Independent samples $t$ test } \\
\hline & $N$ & Mean & SD & $N$ & Mean & SD & \\
\hline Perceived risk & 264 & 23.89 & 19.02 & 399 & 23.89 & 19.02 & $t(661)=-1.74, p=0.08$ \\
\hline Perceived control & 264 & 60.03 & 25.47 & 399 & 61.34 & 24.05 & $t(661)=0.67, p=0.51$ \\
\hline Worry about catching COVID-19 & 265 & 2.39 & 1.04 & 401 & 2.20 & 0.89 & $t(664)=-2.53, p<0.01^{*}$ \\
\hline Severity of illness & 261 & 1.91 & 1.09 & 397 & 1.59 & 0.87 & $t(656)=-4.17, p<0.01^{*}$ \\
\hline Change in physical health & 265 & 2.44 & 0.91 & 401 & 2.61 & 0.91 & $t(664)=2.31, p<0.05^{*}$ \\
\hline Change in mental health & 265 & 1.93 & 1.09 & 401 & 2.08 & 0.91 & $t(664)=1.80, p=0.07$ \\
\hline Exercise & 259 & 1.40 & 1.23 & 393 & 1.74 & 1.24 & $t(649)=3.40, p<0.01^{*}$ \\
\hline Technology use & 264 & 3.37 & 1.21 & 401 & 3.06 & 1.16 & $t(663)=-3.28, p<0.01^{*}$ \\
\hline Sleep & 264 & 14.49 & 5.70 & 401 & 10.15 & 5.62 & $t(662)=-9.72, p<0.01^{*}$ \\
\hline Loneliness & 264 & 5.63 & 2.30 & 401 & 4.56 & 2.44 & $t(663)=-5.61, p<0.01^{*}$ \\
\hline Uncertainty about the future & 265 & 3.38 & 1.13 & 401 & 2.78 & 1.08 & $t(664)=-6.88, p<0.00^{*}$ \\
\hline Psychological distress & 265 & 20.23 & 6.24 & 401 & 16.33 & 6.64 & $t(664)=-7.69, p<0.01^{*}$ \\
\hline Health anxiety & 265 & 5.50 & 2.88 & 400 & 4.10 & 2.66 & $t(662)=-6.40, p<0.01^{*}$ \\
\hline Well-being & 265 & 16.98 & 5.10 & 401 & 20.77 & 5.87 & $t(664)=8.58, p<0.01^{*}$ \\
\hline
\end{tabular}

(see Table 3), and reported lower levels of exercise, great use of technology, poorer sleep, higher levels of loneliness, uncertainty about the future, psychological distress, health anxiety and lower levels of well-being (all $p s<0.05$; see Table 3 for full statistics).

\section{Association between COVID-fears, behaviour, lifestyle factors and mental health}

See Supplementary materials for results. There was a significant positive correlation between fears about contracting COVID-19, and behaviour change, feelings of uncertainty about the future, poor sleep, psychological distress, and health anxiety (all $p s<0.01$ ). Greater exercise was associated with lower levels of screen time, better sleep, lower levels of psychological distress and a greater sense of wellbeing ( $p s<0.01)$. Overall screen time was associated with more psychological distress; however, when screen time was used to connect with friends and family, the relationship with distress was no longer evident, and increased screen time for connection was associated with lower levels of loneliness and higher levels of well-being ( $p s<0.01$ ). Finally, as expected, all the mental health variables were significantly associated, with positive correlations between loneliness, health anxiety and psychological distress, and an inverse association between these variables and well-being being detected.

\section{Discussion}

This study is novel in its assessment of a broad range of variables that relate not only to psychopathology, but also to young people's experiences of COVID-19, the impact on their learning and relationships, as well as a range of lifestyle factors, including sleep, exercise and screen time. To our knowledge, existing adolescent studies focus on the mental health impact of the pandemic on young people (e.g. [12, $18,19])$ or lifestyle factors (e.g. [40]) or worry, concern and behaviour change related to COVID-19 (e.g. [17]), but not these factors collectively. Assessing these variables in the same sample is necessary to form a rich and complete picture of the disruption to young people's lives, mental health and well-being. The benefit of this approach is demonstrated by our findings; for example, we demonstrate, for the first time, that the use of technology for the purposes of social connection (rather than just technology use per se) is associated with a greater sense of well-being and lower levels of loneliness. This important advance in knowledge can help guide the way teenagers can be encouraged to use technology to maintain social connection, when face-to-face interactions are not possible.

The results of our study also showed that adolescents with a self-reported history of depression or anxiety experienced heightened levels of loneliness, greater trouble sleeping, more uncertainty about the future, higher levels of health anxiety, greater psychological distress and lower levels of well-being, relative to those without a depression or anxiety history, in response to the pandemic. The proportion of participants reporting a history of depression or anxiety was comparable to lifetime prevalence estimates for adolescents [41], suggesting respondents were broadly representative of the general population in terms of psychiatric history. This is the first finding to indicate that Australian adolescents with a history of depression or anxiety experience greater levels of psychopathology and disruption to daily life in response to a crisis; in this 
case, the COVID-19 pandemic. Moreover, these results are consistent with findings examining adolescents in Canada [18] and adults in Australia and the US [5, 6], which collectively suggest that a history of mental illness is a vulnerability factor for deterioration in mental health during the pandemic.

There are important implications that follow on from this finding. First, from a practical perspective, the knowledge that challenges, such as a pandemic, lead to an exacerbation of mental illness among those with a history of anxiety and/or depression suggests that psychological treatments for these disorders could incorporate symptom management plans for challenging situations likely to exacerbate symptoms, as a component of therapy. Moreover, an immediate and much needed policy change is needed to expand existing mental health services for young people. There has already been an increased burden on these services [21] and for services to continue to provide care to young people with exacerbations in existing mental health problems, while simultaneously supporting those presenting to services for the first time, an expansion of such services is imperative. Evaluations of the impact of the pandemic on existing services and their response to the crisis (e.g. [42, 43]) may guide such an expansion and inform policy to deal with future periods of increased mental health service need. Similarly, the placement of clinically trained staff into schools to support students either face-to-face when possible, or via telehealth when not, would do much to reduce the burden on external services. Finally, another method to reduce overburdening existing services is to consider the use of effective digital mental health interventions, and blended modalities that combine digital with clinician support, which will likely improve the efficiency with which young people with mental health problems can be supported [44]. In the longer term, a greater focus on the prevention of mental illness and upskilling young people with effective strategies to manage their mental health, especially at times of vulnerability, will have a downstream impact on ensuring services do not become overburdened, particularly during times of crisis. Taken together, the findings reported in this study underscore the need for a proactive mental health response to support young people through this tumultuous and disruptive time in their lives, through changes at both the practice and policy level.

Although respondents had little direct or indirect experience with COVID-19, more than three quarters were worried about contracting the virus, replicating findings with adults [6] and adolescents [15]. Most young people believed they could reduce their risk of contracting COVID-19 and engaged in behaviors to lower their risk (e.g. handwashing and social distancing), in contrast to common portrayals by the media suggesting young people are not compliant with restrictions (e.g. [45]). Our results showed that worry about contracting COVID-19 was associated with greater levels of overall behaviour change, demonstrating a practical benefit of some degree of worry.

This study also sheds light on the disruption and impact of the pandemic on young people's lives. Almost the whole sample (>95\%) had engaged in online learning, and most reported a negative impact. At the time our survey was undertaken, schools and families had to adapt to online learning from home with little-to-no preparation time. While meta-analyses have shown that with optimal delivery and support, online formats can be as effective as face-toface in terms of learning outcomes for adults [46], there is no evidence to suggest this is the case for young people. Indeed, because school education is primarily face-to-face, data about the effectiveness of online schooling is lacking. Online learning requires a greater level of independence, motivation, and discipline than classroom learning, and these are skills which young people may not have fully developed [47].

Participants reported a negative effect of the pandemic on friendships, and feelings of loneliness, which were associated with higher psychological distress and lower well-being. Adolescents are at a crucial stage of development involving the formation of a sense of self and identity through shared interests and values with their peers [48]. Given a lack of social connection has negative consequences on social and cognitive development [9], and loneliness increases the risk of the development of depression and other disorders [12], mental health prevention and intervention efforts need to focus on improving social connection, particularly in areas that have containment measures in place for prolonged periods. Until restrictions are lifted, the use of technology to connect with others might mitigate the potential disruption to adolescents' social needs. With widespread smartphone use [49], it is reassuring that studies have found that core components of quality face-to-face interactions can be replicated online [50]. Consistent with expectations, our study found that nearly three quarters of participants reported increased use of technology to connect with others and this was associated with lower levels of loneliness and a greater sense of well-being. This finding aligns with research from China showing increased smartphone and social media use at the height of the pandemic [16]. Most adolescents in our study reported spending about $4 \mathrm{~h}$ a day connecting with others online. Whether this will mitigate potential longterm consequences of social deprivation associated with lockdowns will need to be addressed by future longitudinal studies.

Most respondents indicated that the pandemic had increased stress levels within their family and half reported an impact on the job of a parent or carer. Viewed in this context, together with adjusting to online learning and the requirement for parents to manage their own professional responsibilities with caring and supporting their child's 
learning, it is not surprising this has been a stressful time for families [51]. This aligns with findings from around the world that are beginning to highlight higher levels of stress and mental ill-health experienced by certain sub-groups of the population, including women, who are more likely to be in caring roles, as well as parents and young people [3, 7]. Despite this increased family stress, $>50 \%$ of adolescents indicated that their relationships with family members had remained unchanged, and an additional $18.7 \%$ reported an improvement; something that has not previously been found in an adolescent sample. It could be the case that in some families, more time at home with family members has some advantages, and feeling more connected to loved ones is a benefit of COVID-19 that has been found in adults [52].

Encouragingly, $>50 \%$ of the adolescents continued with or increased their regular levels of exercise. There are welldocumented links between exercise and reduced risk of depression and anxiety across the lifespan [53] and the relationship between exercise and lower levels of psychological distress and great well-being in our sample is consistent with this broader literature [16]. Given that even the strictest lockdowns in Australia have allowed for up to $1 \mathrm{~h}$ of exercise per day, exercise could be promoted in public health campaigns to prevent deteriorating mental health should the lockdown and period of restrictions continue. Relatedly, a significant proportion of adolescents also reported increased difficulty sleeping, and exercise is one way in which sleep quality and duration can be improved [54], which was supported by our correlational analyses.

Of concern was that about half of the sample reported a worsening in their physical health, since the pandemic began, while $75 \%$ reported a negative effect on their mental health. The worsening of mental health in our sample was markedly consistent to that of a recent adult Australian survey [6], which found that $78 \%$ of their respondents had reported worsened mental health. Overall, adolescents reported greater psychological distress and lower levels of well-being relative to normative data available from population surveys conducted prior to the pandemic [55, 56], with rates of psychological distress indicative of probable mental illness increasing almost twofold from $24.3 \%$ before the pandemic [56], to $48.3 \%$ in this survey. This finding accords with studies from China and Germany showing elevated mental illness in young people [8, 19]. It is important to note that the $\mathrm{K} 6$ is not a diagnostic interview and so without information about the exact duration of the symptoms, degree of interference in daily life and distress to the individual, drawing diagnostic conclusions is not possible. We also cannot rule out the heightened levels of psychological distress as being at least in part attributable to sampling bias, inherent with online surveys. With these limitations in mind, our data nonetheless suggest that there are significantly elevated rates of psychological distress [56], providing insight into the acute effects of the COVID-19 pandemic on adolescent mental health.

There are several study limitations. First, as a convenience sample recruited online due to the ease of rapid administration, $72 \%$ were female, limiting the generalizability of findings to the broader adolescent population. The importance of sampling approach has been noted as a key concern during COVID-19 [25]. Follow-up studies should use diagnostic assessments, to provide an independent assessment of mental health. The study was cross sectional and so causal conclusions cannot be drawn.

Despite these limitations, this study has provided insight into the impact of the COVID-19 pandemic on multiple facets of young people's lives. Marked deterioration in the mental health of adolescents, evidenced by three quarters of respondents reporting a negative impact of the pandemic on mental health and almost half reporting psychological distress levels indicative of a probable mental illness (a twofold increase from pre-pandemic levels), emphasises the need for adequate infrastructure to support the mental health and recovery of this already vulnerable population. This study showed worse outcomes for those with pre-existing mental health conditions, further indicating the need for adequate services during times of crisis, but also the need for current treatments to provide young people with skills to manage their mental health during times of adversity.

Supplementary Information The online version contains supplementary material available at https://doi.org/10.1007/s00787-021-01790-x.

Acknowledgements Sincere thanks to all the study participants for taking part in this survey. Thanks also to Iana Wong for assisting with Qualtrics programming, to Dr Samantha Spanos for survey testing and to Dr Lyndsay Brown for providing feedback on the manuscript.

Author contributions All authors contributed to the study conception and design. Material preparation, data collection and analysis was performed by AWS. The first draft of the manuscript was written by AWS, with support from SHL and JRB. All authors commented on previous versions of the manuscript. All authors read and approved the final manuscript.

Funding This study was supported by the Black Dog Institute, a NSW Health Fellowship awarded to AW-S and a MRFF Career Development Fellowship awarded to JN. The funders had no role in the study design, collection, analysis or interpretation of the data, writing the manuscript, or the decision to submit the paper for publication.

Availability of data and material Not publicly available due to the sensitive nature of the data and ethical guidelines.

\section{Declarations}

Conflict of interest None to declare. 
Open Access This article is licensed under a Creative Commons Attribution 4.0 International License, which permits use, sharing, adaptation, distribution and reproduction in any medium or format, as long as you give appropriate credit to the original author(s) and the source, provide a link to the Creative Commons licence, and indicate if changes were made. The images or other third party material in this article are included in the article's Creative Commons licence, unless indicated otherwise in a credit line to the material. If material is not included in the article's Creative Commons licence and your intended use is not permitted by statutory regulation or exceeds the permitted use, you will need to obtain permission directly from the copyright holder. To view a copy of this licence, visit http://creativecommons.org/licenses/by/4.0/.

\section{References}

1. World Health Organization (2020) WHO Coronavirus Disease (COVID-19) dashboard. World Health Organization. https:// covid19. who.int/. Published 2020. Accessed 03 Aug 2020

2. Wang $C$ et al (2020) A longitudinal study on the mental health of general population during the COVID-19 epidemic in China. Brain Behav Immun 87:40-48

3. Pierce $M$ et al (2020) Mental health before and during the COVID-19 pandemic: a longitudinal probability sample survey of the UK population. Lancet Psychiatry. https://doi.org/ 10.1016/S2215-0366(20)30308-4

4. Rodriguez-Rey R, Garrido-Hernansaiz H, Collado S (2020) Psychological impact of COVID-19 in Spain: early data report. Psychol Trauma Theory Res Pract Policy. https://doi.org/10. 1037/tra0000943

5. Van Rheenen TE et al (2020) Mental health status of individuals with a mood-disorder during the COVID-19 pandemic in Australia: initial results from the COLLATE project. J Affect Disord 275:69-77

6. Newby JM et al (2020) Acute mental health responses during the COVID-19 pandemic in Australia. PLoS One 15(7):e0236562

7. Rossell SL et al (2021) An overview of current mental health in the general population of Australia during the COVID-19 pandemic: results from the COLLATE project. Psychiatry Res 296:113660

8. Racine N et al (2020) Child and adolescent mental illness during COVID-19: a rapid review. Psychiatry Res 292:113307-113307

9. Orben A, Tomova L, Blakemore S-J (2020) The effects of social deprivation on adolescent development and mental health. Lancet Child Adolesc Health 4(8):634-640

10. Parker JG et al (1999) Dimensions of children's friendship adjustment: implications for understanding loneliness. In: Rotenberg KJ, Hymel S (eds) Loneliness in childhood and adolescence. Cambridge University Press, Cambridge, pp 201-222

11. Sebastian CL et al (2011) Developmental influences on the neural bases of responses to social rejection: implications of social neuroscience for education. Neuroimage 57(3):686-694

12. Loades ME et al (2020) Rapid systematic review: the impact of social isolation and loneliness on the mental health of children and adolescents in the context of COVID-19. J Am Acad Child Adolesc Psychiatry. https://doi.org/10.1016/j.jaac.2020.05.009

13. Racine N, Korczak DJ, Madigan S (2020) Evidence suggests children are being left behind in COVID-19 mental health research. Eur Child Adolesc Psychiatry. https://doi.org/10.1007/ s00787-020-01672-8

14. Zhou S-J et al (2020) Prevalence and socio-demographic correlates of psychological health problems in Chinese adolescents during the outbreak of COVID-19. Eur Child Adolesc Psychiatry. https://doi.org/10.1007/s00787-020-01541-4
15. Duan L et al (2020) An investigation of mental health status of children and adolescents in china during the outbreak of COVID-19. J Affect Disord 275:112-118

16. Chen $\mathrm{F}$ et al (2020) Depression and anxiety among adolescents during COVID-19: a cross-sectional study. Brain Behav Immun. https://doi.org/10.1016/j.bbi.2020.05.061

17. Oosterhoff B et al (2020) Adolescents' motivations to engage in social distancing during the COVID-19 pandemic: associations with mental and social health. J Adolesc Health. https://doi.org/ 10.1016/j.jadohealth.2020.05.004

18. Cost KT et al (2021) Mostly worse, occasionally better: impact of COVID-19 pandemic on the mental health of Canadian children and adolescents. Eur Child Adolesc Psychiatry. https://doi. org/10.1007/s00787-021-01744-3

19. Ravens-Sieberer U et al (2021) Impact of the COVID-19 pandemic on quality of life and mental health in children and adolescents in Germany. Eur Child Adolesc Psychiatry. https://doi. org/10.1007/s00787-021-01726-5

20. Clayton R (2020) Statistics show increase in children presenting to hospitals after self-harming. ABC News 2020. https://www. abc.net.au/news/2020-08-08/young-people-self-harming-endup-in-hospital-emergency-rooms/12532040 Accessed 20 Aug 2020

21. Young E (2020) Coronavirus worries have Australian children calling Kids Helpline every 69 seconds. SBS News 2020. https://www.sbs.com.au/news/coronavirus-worries-have-austr alian-children-calling-kids-helpline-every-69-seconds Accessed 01 Aug 2020

22. YoungMinds (2020) Coronavirus: impact on young people with mental health needs. UK 2020. https://youngminds.org. uk/media/3904/coronavirus-report-summer-2020-final.pdf Accessed 01 Aug 2020

23. Wade M, Prime H, Browne DT (2020) Why we need longitudinal mental health research with children and youth during (and after) the COVID-19 pandemic. Psychiatry Res 290:113143

24. Holmes EA et al (2020) Multidisciplinary research priorities for the COVID-19 pandemic: a call for action for mental health science. Lancet Psychiatry 7(6):547-560

25. Pierce $M$ et al (2020) Says who? The significance of sampling in mental health surveys during COVID-19. Lancet Psychiatry 7(7):567-568

26. Spriggs $M$ (2010) Understanding consent in research involving children: a handbook for Human Research Ethics Committees and researchers. University of Melbourne, Melbourne

27. Idler EL, Benyamini Y (1997) Self-rated health and mortality: a review of twenty-seven community studies. J Health Soc Behav 38(1):21-37

28. Faasse K, Newby J (2020) Public perceptions of COVID-19 in Australia: perceived risk, knowledge, health-protective behaviors, and vaccine intentions. Front Psychol 11:551004-551004

29. Bastien CH, Vallières A, Morin CM (2001) Validation of the Insomnia Severity Index as an outcome measure for insomnia research. Sleep Med 2(4):297-307

30. Chung KF, Kan KK, Yeung WF (2011) Assessing insomnia in adolescents: comparison of Insomnia Severity Index, Athens Insomnia Scale and Sleep Quality Index. Sleep Med 12(5):463-470

31. Russell DW (1996) UCLA Loneliness Scale (version 3): reliability, validity, and factor structure. J Pers Assess 66(1):20-40

32. Kessler RC et al (2002) Short screening scales to monitor population prevalences and trends in non-specific psychological distress. Psychol Med 32(6):959-976

33. Kessler RC et al (2003) Screening for serious mental illness in the general population. Arch Gen Psychiatry 60(2):184-189

34. Furukawa TA et al (2003) The performance of the K6 and K10 screening scales for psychological distress in the Australian 
National Survey of Mental Health and Well-Being. Psychol Med 33(2):357-362

35. Ferro MA (2019) The psychometric properties of the Kessler Psychological Distress Scale (K6) in an epidemiological sample of Canadian youth. The Canadian Journal of Psychiatry 64(9):647-657

36. Tennant R et al (2007) The Warwick-Edinburgh Mental Wellbeing Scale (WEMWBS): development and UK validation. Health Qual Life Outcomes 5(1):63

37. McKay MT, Andretta JR (2017) Evidence for the psychometric validity, internal consistency and measurement invariance of Warwick Edinburgh Mental Well-being Scale scores in Scottish and Irish adolescents. Psychiatry Res 255:382-386

38. Weck F, Bleichhardt G, Hiller W (2010) Screening for hypochondriasis with the illness attitude scales. J Pers Assess 92(3):260-268

39. Lawrence D et al (2015) The mental health of children and adolescents: report on the second Australian child and adolescent survey of mental health and wellbeing

40. Xiang M, Zhang Z, Kuwahara K (2020) Impact of COVID-19 pandemic on children and adolescents' lifestyle behavior larger than expected. Prog Cardiovasc Dis 63(4):531-532. https://doi. org/10.1016/j.pcad.2020.04.013

41. Merikangas KR et al (2010) Prevalence and treatment of mental disorders among US children in the 2001-2004 NHANES. Pediatrics 125(1):75-81

42. Cui Y, Li Y, Zheng Y (2020) Mental health services for children in China during the COVID-19 pandemic: results of an expert-based national survey among child and adolescent psychiatric hospitals. Eur Child Adolesc Psychiatry 29(6):743-748

43. Revet A et al (2020) ESCAP CovCAP survey of heads of academic departments to assess the perceived initial (April/May 2020) impact of the COVID-19 pandemic on child and adolescent psychiatry services. Eur Child Adolesc Psychiatry. https://doi.org/ 10.1007/s00787-020-01699-x

44. Garrido S et al (2019) What works and what doesn't work? A systematic review of digital mental health interventions for depression and anxiety in young people. Front Psych 10:759-759

45. Carmody J (2020) Young people hit back at coronavirus criticism that they don't understand social distancing and COVID-19 risk. ABC news 2020. https://www.abc.net.au/news/2020-03-24/youngpeople-hit-back-on-social-distancing-coronavirus-rules/12081526 Accessed 16 Jun 2020
46. Jahng $\mathrm{N}$ et al (2007) Student achievement in online distance education compared to face-to-face education. Eur J Open Distance E-Learn 10

47. Blakemore SJ, Choudhury S (2006) Development of the adolescent brain: implications for executive function and social cognition. J Child Psychol Psychiatry 47(3-4):296-312

48. Pfeifer JH, Berkman ET (2018) The development of self and identity in adolescence: neural evidence and implications for a value-based choice perspective on motivated behavior. Child Dev Perspect 12(3):158-164

49. Pew Research Centre (2019, February 5). Smartphone owernership is growing rapidly around the world, but not always equally [Press release] https://www.pewresearch.org/global/2019/02/05/ smartphone-ownership-is-growing-rapidly-around-the-world-butnot-always-equally/

50. Yau JC, Reich SM (2018) Are the qualities of adolescents' offline friendships present in digital interactions? Adolesc Res Rev 3(3):339-355

51. Thorell LB et al (2021) Parental experiences of homeschooling during the COVID-19 pandemic: differences between seven European countries and between children with and without mental health conditions. Eur Child Adolesc Psychiatry. https://doi.org/ 10.1007/s00787-020-01706-1

52. Stech EP, Tang S, Wong I, O'Moore K, Newby JM Proactive coping and silver linings during the COVID-19 pandemic. [submitted] Black Dog Institute, The University of New South Wales.

53. Biddle SJH, Asare M (2011) Physical activity and mental health in children and adolescents: a review of reviews. Br J Sports Med 45(11):886

54. Dolezal B et al (2017) Interrelationship between sleep and exercise: a systematic review. Adv In Prev Med 27:1364387

55. Ng Fat L et al (2017) Evaluating and establishing national norms for mental wellbeing using the short Warwick-Edinburgh Mental Well-being Scale (SWEMWBS): findings from the Health Survey for England. Qual Life Res 26(5):1129-1144

56. Hall S, Fildes J, Perrens B, et al (2019) Can we talk? Seven year youth mental health report 2012-2018. https://www.blackdogin stitute.org.au/wp-content/uploads/2020/04/2017-youth-mentalhealth-report_mission-australia-and-black-dog-institute.pdf? sfvrsn=6 Accessed 01 Aug 2020 